\title{
Transfemoral transcatheter aortic valve implantation in a patient with a severe aortic stenosis and cardiogenic shock requiring intra-aortic balloon pump support
}

\author{
Piotr Chodór ${ }^{1}$, Krzysztof Wilczek², Roman Przybylski ${ }^{3}$, Andrzej Świątkowski ${ }^{1}$, Jan Głowacki ${ }^{4}$, \\ Zbigniew Kalarus ${ }^{1}$, Marian Zembala ${ }^{3}$ \\ ${ }^{1}$ Department of Cardiology, Congenital Heart Diseases and Electrotherapy, Medical University of Silesia in Katowice, Silesian Center \\ for Heart Diseases in Zabrze, Poland \\ ${ }^{2} 3^{\text {rd }}$ Department of Cardiology, Medical University of Silesia in Katowice, Silesian Center for Heart Diseases in Zabrze, Poland \\ ${ }^{3}$ Department of Cardiac Surgery and Transplantology, Medical University of Silesia in Katowice, Silesian Center for Heart Diseases \\ in Zabrze, Poland \\ ${ }^{4}$ Department of Radiology, Medical University of Silesia in Katowice, Silesian Center for Heart Diseases in Zabrze, Poland
}

Postep Kardiol Inter 2015; 11, 1 (39): 55-57 DOI: $10.5114 /$ pwki.2015.49187

\begin{abstract}
A b s tract
The following paper presents a patient with severe aortic stenosis and severely reduced left ventricular ejection fraction with intra-aortic balloon pump counterpulsation support, who underwent transfemoral aortic valve implantation of a CoreValve prosthesis.
\end{abstract}

Key words: transcatheter aortic valve implantation, cardiogenic shock, CoreValve.

\section{Introduction}

Aortic valve replacement (AVR) is the treatment of choice for severe aortic stenosis (AS). However, many patients with indications for AVR do not undergo surgery due to high operative risk [1]. Therefore, transcatheter aortic valve implantation (TAVI) becomes a standard procedure in high-risk patients. CoreValve (Medtronic Minneapolis, MN, USA) and Edwards SAPIEN (Edwards Lifesciences Inc., Irvine, CA, USA) prostheses are widely used for transcatheter application [2, 3]. In the majority of cases these valves are implanted in haemodynamically stable patients.

In this case study, we describe a patient with a severe AS in cardiogenic shock (CS), which required intra-aortic balloon pump (IABP) support, also recovering from sepsis, who underwent TAVI of a Medtronic CoreValve prosthesis (MCV).

\section{Case report}

A 79-year-old male was admitted to the cardiac care unit (CCU) with symptoms of acute heart failure (HF) in the course of severe AS, evolving to pulmonary oedema and CS. Due to the rapid progression of HF, in addition to dopamine infusion, an IABP support was started. Echocardiography $(\mathrm{ECHO})$ demonstrated the following: left ventricle (LV) $6.6 / 5.6 \mathrm{~cm}$, ascending aorta (Ao) $4.1 \mathrm{~cm}$, left atrium (LA) $5.2 \mathrm{~cm}$, left ventricular ejection fraction (LVEF) of $10 \%$ on admission and $21 \%$ after an IABP introduction, and moderate/severe mitral regurgitation (MR). Peak and mean AV gradient was $137 \mathrm{~mm} \mathrm{Hg}$ and $65 \mathrm{~mm} \mathrm{Hg}$, respectively, with a mild aortic regurgitation (AR). Calcifications were found in the AV. The AV annulus was $2.5 \mathrm{~cm}$. Moderate tricuspid regurgitation (TR) was present with a right ventricle systolic pressure (RVSP) of $70 \mathrm{~mm} \mathrm{Hg}$. It was decided to perform an emergency balloon aortic valvuloplasty (BAV). During the BAV, the IABP was set at a $1: 1$ assist ratio, but it was turned off during the RV rapid pacing (RP). After the BAV the mean AV gradient decreased from $57 \mathrm{~mm} \mathrm{Hg}$ to $27 \mathrm{~mm} \mathrm{Hg}$ and the AV area increased from $0.35 \mathrm{~cm}^{2}$ to $0.7 \mathrm{~cm}^{2}$. The baseline NT proBNP was over $35000 \mathrm{pg} / \mathrm{ml}$. Substantial improvement of the patient's state allowed reduction of catecholamines and weaning off the IABP on the $16^{\text {th }}$ day after admis-

Corresponding author:

Piotr Chodór MD, Department of Cardiology, Congenital Heart Diseases and Electrotherapy, Medical University of Silesia in Katowice,

Silesian Center for Heart Diseases in Zabrze, 2 Szpitalna St, 41-800 Zabrze, Poland, phone: +48 $322735356,+48660442582$,

e-mail: chodor_piotr@go2.pl

Received: 26.06.2014, accepted: 8.01.2015. 
sion. However, 4 days later a re-introduction of IABP was required due to haemodynamic deterioration. Coronary angiography revealed normal coronary arteries. The computed tomography (CT) angiography parameters were as follows: annulus diameter $2.6 \mathrm{~cm}$, aortic root $3.1 \mathrm{~cm}$, sinotubular junction $2.7 \mathrm{~cm}$, ascending aorta $4.6 \mathrm{~cm}$; femoral and subclavian arteries $>0.6 \mathrm{~cm}$. The Logistic Euroscore and STS score were $30 \%$ and $12 \%$, respectively. Due to the high risk, the patient was considered a candidate for TAVI. The patient displayed symptoms of infection, and as a result Acinetobacter Baumani was isolated from blood cultures. The infection was treated with antibiotics according to the antibiogram. Despite the initial improvement, there was a recurrence of fever. Fungal infection was suspected and so caspofungin was started. The TAVI procedure had to be postponed several times due to observed symptoms of infection. The medical team decided to perform TAVI because they were convinced that further delay of the intervention would result in subsequent deterioration of the patient's condition and ultimately death. The TAVI was performed 3 weeks after the IABP re-implantation when the patient had been without fever for 5 consecutive days and a significant drop of inflammatory parameters was recorded. Blood cultures, however, were still positive on the day prior to the procedure. The femoral approach was chosen. A surgical exposure and cut-down of the artery was applied in deep sedation and local anaesthesia. The patient was IABP-dependent throughout the procedure. The patient's blood pressure was 90/41 mean $64 \mathrm{~mm}$ $\mathrm{Hg}$ with IABP support. The BAV was performed on RP at a rate of $190 \mathrm{~min}^{-1}$ and on IABP switched to 'standby' mode. Immediately after BAV no increase in the AR

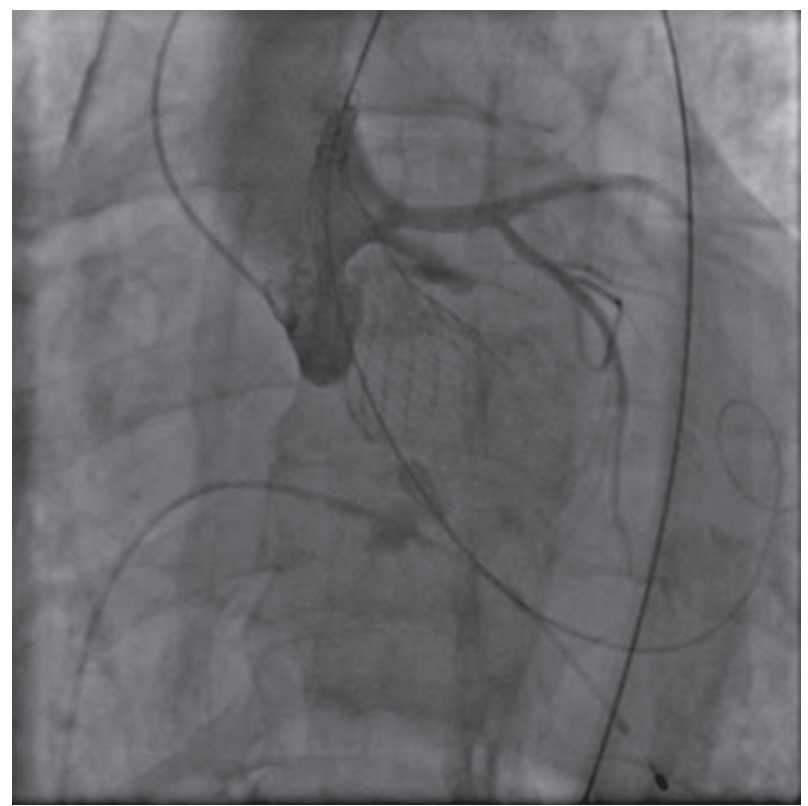

Figure 1. Aortography - aortic valve implantation during ventricular fibrillation was detected on ECHO. A MCV size 29 was chosen. Frequent contrast injection not only made positioning of the valve easier but also ensured that no significant AR was present. During the implantation when $2 / 3$ of MCV was deployed and the valve started to function, ventricular fibrillation occurred. After checking the position of the valve, the implantation was completed promptly and cardiac massage was started followed by five defibrillation shocks delivered with sinus rhythm restoration (Figure 1). The patient was intubated and ventilated. The procedure was completed under general anaesthesia.

The patient was weaned off the ventilator and the IABP on the $2^{\text {nd }}$ day after the TAVI. ECHO showed an increase in LVEF to $30 \%$, the peak and mean aortic valve gradient was $21 \mathrm{~mm} \mathrm{Hg}$ and $10 \mathrm{~mm} \mathrm{Hg}$, respectively. Moderate paravalvular leak, moderate MR, and mild TR was noted. No lesions indicative of infective endocarditis were seen on the valves.

In the 6-minute walk test performed prior to hospital discharge the patient was able to cover a distance of $100 \mathrm{~m}$, and after 2 months the distance increased to $340 \mathrm{~m}$. NTpro BNP levels further decreased from $1410 \mathrm{pg} / \mathrm{ml}$ at hospital discharge to $1204 \mathrm{pg} / \mathrm{ml}$ after 2 months. The LVEF increased to 39\%; peak and mean AV gradients were $19 \mathrm{~mm}$ $\mathrm{Hg}$ and $11 \mathrm{~mm} \mathrm{Hg}$, respectively. Mild paravalvular leak, and trivial $M R$ and $T R$ were present.

In the 6-minute walk test performed after 2 years the patient covered a distance of $378 \mathrm{~m}$. NT-pro BNP level was $1608 \mathrm{pg} / \mathrm{ml}$. The ECHO showed LVEF 57\%, and peak and mean aortic valve gradient were $23 \mathrm{~mm} \mathrm{Hg}$ and $13 \mathrm{~mm} \mathrm{Hg}$, respectively. Trivial paravalvular leak, mild $M R$, and trivial TR were noted. No lesions indicative of infective endocarditis were seen on the valves.

\section{Discussion}

Performing TAVI in haemodynamically unstable patients is risky. Relevant published data are quite scarce, apart from the first-in-man TAVI report by Cribier et al. [2]. They implanted a balloon-expandable valve in a patient with CS having a low LVEF $<20 \%$, without any mechanical circulatory assist. Haemodynamically unstable patients requiring $\mathrm{IABP}$ assist usually undergo BAV in order to obtain temporary clinical improvement with LVEF increase. The authors performed BAV in haemodynamically unstable patients with end-stage AS. In cases where IABP support is used during BAV, it is switched to 'standby' mode at the time of the RP and balloon inflation. Support is restarted if no significant aortic regurgitation appears on echocardiography. Valve implantation (AVR or TAVI) is usually carried out after the patient becomes stable. Valve implantation is not recommended in patients with an LVEF lower than $20 \%$. Low LVEF is not a contraindication to conventional surgery if the left ventricle generates a mean gradient of more than $40 \mathrm{~mm} \mathrm{Hg}$, despite its impaired contractility [4]. In the presented 
case the aortic valve gradient was continuously high despite an LVEF value of about $10 \%$ at admission. No other possible causes for decreased LVEF apart from aortic stenosis were found. Therefore, it was decided to perform BAV followed by TAVI. Another reason for the delay of TAVI was the fact that the patient had sepsis, so the implanted valve prosthesis would be exposed to a high risk of prosthetic valve endocarditis.

Due to a lack of improvement and the need for IABP re-implantation, the TAVI procedure was performed during normal IABP support. The timing of the intervention was dictated by the worsening clinical status, including the onset of depression. Despite the high operative risk, the Heart Team decided that further delay of the intervention would result in haemodynamic breakdown and subsequent death of the patient. The patient was informed about the high operative risk and gave his consent for the procedure. During the TAVI procedure BAV was performed again to avoid any problems with the delivery and the positioning of the valve in this particular patient. The potential risk of creating acute aortic regurgitation was low since the same balloon size was used, as in the first BAV. Performing BAV was considered to be a safer option. Kahlert et al. demonstrated that valve positioning and deployment is associated with more cerebral embolic events than BAV $[4,5]$. An important issue is circulatory support in a patient with severely depressed LV function. A few options could be taken into consideration. The IABP was chosen in this case, which was very effective when administered on admission because it stabilised the patient and it is believed that it made the BAV procedure safe and without complications. To use a ventricular assist device like the 'TandemHeart' could have been another option to increase the safety of the TAVI procedure in this case. The 'TandemHeart' device provides even better LV support, but it is associated with an increased rate of bleeding complications and, similarly to IABP, there is no documented experience of its use in TAVI $[5,6]$. Transapical implantation with extracorporeal circulation has been excluded due to the very low EF, but its successful use in patients with CS has been reported recently by D'Ancona et al. [6, 7]. The less invasive nature of IABP made it a preferable method and it was planned for the TAVI procedure via the right femoral artery approach. This was achieved by surgical dissection of the right femoral artery during deep sedation and under a local anaesthesia. The presented case reveals that in a group of critically unstable patients TAVI can be safely performed during circulatory support with IABP. In such cases the use of general anaesthesia should be considered for the entire procedure.

\section{Conclusions}

Patients with critical AS complicated by IABP-dependent CS, with severely reduced LVEF, are still candidates for TAVI, which can be successfully performed.
Ventricular fibrillation requiring resuscitation may occur during valve implantation in those patients. The presence of an operating IABP balloon does not interfere with MCV implantation via transfemoral approach. Good clinical and echocardiographic results were observed in long-term follow up, with improved ejection fraction of LV and a stable mean gradient.

\section{Conflict of interest}

The authors declare no conflict of interest.

\section{References}

1. Iung B, Cachier A, Baron G, et al. Decision-making in elderly patients with severe aortic stenosis: why are so many denied surgery? Eur Heart J 2005; 26: 2714-20.

2. Cribier A, Eltchaninoff $\mathrm{H}$, Bash A, et al. Percutaneous transcatheter implantation of an aortic valve prosthesis for calcific aortic stenosis: first human case description. Circulation 2002; 106: 3006-8.

3. Grube E, Buellesfeld L, Mueller R, et al. Progress and current status of percutaneous aortic valve replacement: results of three device generations of the CoreValve Revalving system. Circ Cardiovasc Interv 2008; 1: 167-75.

4. Vahanian A, Baumgartner H, Bax J, et al. Guidelines on the management of valvular heart disease: The Task Force on the Management of Valvular Heart Disease of the European Society of Cardiology. Eur Heart J 2007; 28: 230-68.

5. Kahlert P, Al-Rashid F, Döttger P, et al. Cerebral embolization during transcatheter aortic valve implantation: a transcranial Doppler study. Circulation 2012; 126: 1245-55.

6. Tanaka K, Rangarajan K, Azarbal B, et al. Percutaneous ventricular assist during aortic valvuloplasty: potential application to the deployment of aortic stent-valves. Tex Heart Inst J 2007; 34: 36-40.

7. D’Ancona G, Pasic M, Buz S, et al. Transapical transcatheter aortic valve replacement in patients with cardiogenic shock. Interact Cardiovasc Thorac Surg 2012; 14: 426-30. 